\title{
High Frequency Scattering by a Smooth Coated Cylinder Simulated with Generalized Impedance Boundary Conditions
}

\author{
Hasnain H. Syed and John L. Volakis
}

\author{
Radiation Laboratory \\ Department of Electrical Engineering and Computer Science \\ The University of Michigan \\ Ann Arbor, Michigan 48109-2122
}

\begin{abstract}
Rigorous UTD (Uniform Geometrical Theory of Diffraction) diffraction coefficients are presented for a convex coated cylinder simulated with generalized impedance boundary conditions. In particular, ray solutions are obtained which remain valid in the transition region and reduce uniformly to those in the deep lit and shadow regions. These involve new transition functions in place of the normal Fock-type integrals characteristic to the impedance cylinder. Also, a uniform asymptotic solution is obtained for observations in the close vicinity of the cylinder. As usual, the diffraction coefficients for the coated convex cylinder are obtained upon a generalization of the correpsonding coefficients for the circular coated cylinder.
\end{abstract}


- nom

INRQ471 


\section{Introduction}

The problem of scattering by a smooth convex impedance cylinder has received much attention. Wang [1, 2] presented ray-optical solutions for the impedance and coated cylinders. His results are valid only in the deep lit and shadow regions and do not apply to the case where the observation point is in the transition region. Wait and Conda $[3,4]$ developed a solution which is valid in the transition region and for observation points on and off the surface. However, as pointed out by Pathak [5] it did not uniformly reduce to the ray solution $[6,7]$ exterior to the transition regions. Also, it is not valid on the portion of the surface in the transition region and these limitations were the primary motivations of Pathak's work [5] for the perfectly conducting convex cylinder. Recently, Kim and Wang [8] presented a solution applicable to a coated cylinder that remained valid in the transition region. They employed a heuristic approach to obtain the numerical values of the resulting transition integral applicable to a coated cylinder. Their solution is uniform but is not applicable to the close vicinity of the cylinder.

In this paper we present a rigorous UTD solution of the diffraction by a coated cylinder simulated with generalized impedance boundary conditions In addition, a uniform asymptotic solution is obtained which remains valid when the observation point is in the close vicinity of the cylinder. An important aspect of the paper is also the use of second order generalized impedance boundary conditions (GIBC) for the simulation of the coating. Their derivation has already been given in [9] and [10] and are characterized by the inclusion of higher order field derivatives in their definition. Because of this they are less local which leads to an improved simulation (with respect to the standard impedance boundary condition - SIBC) of the coating in a manner analogous to the order of the highest derivative kept in the condition. Recently, they were successfully applied to a number of diffraction problems [11], [12] and have also been used in numerical simulations of multilayer coatings (see fig. 1) [13]. These applications provided a measure of the accuracy of the proposed GIBC and in particular accuracy criteria were derived in [13] for the second order conditions as a function of coating thickness and composition.

The UTD solution to be presented here parallels that given by Pathak 
[5] for the circular perfectly conducting cylinder. However, in the case of the coated cylinder the resulting UTD expressions are in terms of Fock-type functions whose efficient evaluation is of primary concern. In the following we first present the eigenfunction solution based on the second order GIBC simulation of a circular coated cylinder. By employing Watson's transformation this is written in integral form which is then cast in a ray representation. The ray solution is subsequently generalized to the case of a general convex cylinder. Finally, the evaluation of the Fock-type functions is discussed and some results are presented which validate the accuracy of the GIBC eigenfunction and ray solutions. In the process, we demonstrate the improved accuracy of the GIBC solution over the corresponding SIBC solution, and it is also shown how the presented UTD solution can be extended to treat multilayered coated cylinders.

\section{Boundary Conditions}

Consider the coated circular cylinder shown in figure 2(a). We propose to simulate the cylinder with an equivalent one (see fig. 2(b)) satisfying the boundary conditions (an $e^{j w t}$ time convention is assumed throughout) [13]

$$
E_{\phi}=-\frac{\left(a_{2}+a_{o}\right)}{a_{1}} Z H_{z}-\frac{a_{2} Z}{k^{2} b^{2} a_{1}} \frac{\partial^{2} H_{z}}{\partial \phi^{2}}
$$

for $H_{z}$-incidence or

$$
E_{z}=\frac{\left(a_{2}+a_{o}\right)}{a_{1}} Z H_{\phi}-\frac{a_{2}^{\prime}}{k^{2} b^{2}\left(a_{2}^{\prime}+a_{o}^{\prime}\right)} \frac{\partial^{2} E_{z}}{\partial \phi^{2}}
$$

in the case of $E_{z}$-incidence. In these $Z=\frac{1}{Y}$ is the free space intrinsic impedance, $k=\frac{2 \pi}{\lambda}$ is the free space wave number, and $a_{m}$ with $a_{m}^{\prime}$ are constants which are dependent on the material properties of the coating.

Assuming a single layer coating of thickness $\delta$ and denoting the associated relative constitutive parameters as $\epsilon_{r}$ and $\mu_{r}$, we have

$$
\begin{aligned}
& a_{o}=\left(\frac{\epsilon_{r} \mu_{r}-1}{\epsilon_{r}}\right) k \delta \\
& a_{1}=-j
\end{aligned}
$$




$$
a_{2}=k \delta / \epsilon_{\tau}
$$

and

$$
\begin{aligned}
& a_{o}^{\prime}=1 \\
& a_{1}^{\prime}=+j k \mu_{r} \delta \\
& a_{2}^{\prime} \approx 0
\end{aligned}
$$

These constants are valid for $\sqrt{\epsilon_{r} \mu_{r}} \delta \lesssim 0.15 \lambda$ and for higher contrast coating they must be redefined as [9]

$$
\begin{aligned}
a_{o} & =\left(N-\frac{1}{2 N}\right)\left[\tan (k \delta N)-\tan \left(\frac{k \delta}{2 N}\right)\right] \\
a_{1} & =-j \epsilon_{r}\left[1+\tan (k \delta N) \tan \left(\frac{k \delta}{2 N}\right)\right] \\
a_{2} & =\frac{1}{2 N}\left[\tan (k \delta N)-\tan \left(\frac{k \delta}{2 N}\right)+k \delta\left(N-\frac{1}{2 N}\right)\right. \\
& \left.\cdot\left\{1+\tan (k \delta N) \tan \left(\frac{k \delta}{2 N}\right)\right\}\right] \\
a_{0}^{\prime}= & \left(N-\frac{1}{2 N}\right)\left[1+\tan (k \delta N) \tan \left(\frac{k \delta}{2 N}\right)\right] \\
a_{1}^{\prime}= & j \mu\left[\tan (k \delta N)-\tan \left(\frac{k \delta}{2 N}\right)\right] \\
a_{2}^{\prime}= & \frac{1}{2 N}\left[1+\tan (k \delta N) \tan \left(\frac{k \delta}{2 N}\right)-k \delta\left(N-\frac{1}{2 N}\right)\right. \\
. & \left.\left\{\tan (k \delta N)-\tan \left(\frac{k \delta}{2 N}\right)\right\}\right]
\end{aligned}
$$


We note that (1) and (2) reduce to the standard impedance boundary conditions (SIBC) when $a_{2}$ and $a_{2}^{\prime}$ are set to zero and

$$
\eta=\frac{a_{o}}{a_{1}}=\frac{a_{1}^{\prime}}{a_{o}^{\prime}}
$$

where $\eta=-j \sqrt{\frac{\mu_{r}}{\epsilon_{r}}} \tan \left(k \sqrt{\epsilon_{r} \mu_{r}} \delta\right)$ is the normalized impedance of the coating. We also remark that $a_{m}$ and $a_{m}^{\prime}$ satisfy the duality relation

$$
\frac{a_{1}}{a_{2}+a_{o}}=\frac{a_{2}^{\prime}+a_{o}^{\prime}}{a_{1}^{\prime}}
$$

The aforementioned boundary conditions can be employed for simulating multilayer coating by simply redefining the material constants $a_{m}$ and $a_{m}^{\prime}$ as discussed, for example, in [10] and [13]

\section{Eigenfunction and Integral representa- tion}

Consider the plane wave

$$
u^{i}=u_{o} e^{j k x}=u_{o} e^{j k \rho \cos \phi}
$$

incident on the coated circular cylinder shown in figure 2, where $u_{o}$ is the amplitude of the wave and $u^{i}$ denotes either the $E_{z}$ or $H_{z}$ component of the incident field. An eigenfunction representation of the total field in the presence of the cylinder can be generally written as

$$
u^{t}=\sum_{n=-\infty}^{\infty} j^{n}\left[J_{n}(k \rho)+A_{n} H_{n}^{(2)}(k \rho)\right] e^{-j n \phi}
$$

where $J_{n}(\cdot)$ is the Bessel function of order $n$ and $H_{n}^{(2)}(\cdot)$ denotes the $n$th order Hankel function of the second kind. To find the constant $A_{n}$ we enforce the GIBC given by (1) or (2) at $\rho=b$. This yields

$$
A_{n}=-\frac{J_{n}^{\prime}(k b)+Q(n) J_{n}(k b)}{H_{n}^{(2)}(k b)+Q(n) H_{n}^{(2)}(k b)}
$$


in which the prime denotes differentiation with respect to the Bessel or Hankel function argument,

$$
Q(n)= \begin{cases}-j\left(\frac{a_{2}+a_{0}}{a_{1}}-n^{2} \frac{a_{2}}{k^{2} b^{2} a_{1}}\right) & \text { for } H_{z} \text {-incidence } \\ -j\left(\frac{a_{2}^{\prime}+a_{o}^{\prime}}{a_{1}^{\prime}}-n^{2} \frac{a_{0}^{\prime}}{k^{2} b^{2} a_{1}^{\prime}}\right) & \text { for } E_{z} \text {-incidence }\end{cases}
$$

and when $a_{2}=a_{2}^{\prime}=0, Q(n)$ reduces to

$$
Q(n)=Q= \begin{cases}-j \frac{a_{a}}{a_{1}}=-j \eta & \text { for } H_{z} \text {-incidence } \\ -j \frac{a_{o}^{\prime}}{a_{1}^{\prime}}=-j \frac{1}{\eta} & \text { for } E_{z} \text {-incidence }\end{cases}
$$

To obtain a ray representation of (10) in conjunction with (12) we must first recast $u^{t}$ in integral form. Employing the usual Watson's transformation [14] and noting that $Q(n)=Q(-n)$, we find [5]

$$
u^{t}=u_{1}+u_{2}
$$

where

$$
u_{1}=u_{0} \int_{-\infty}^{\infty}\left[J_{\nu}(k \rho)-\frac{J_{\nu}^{\prime}(k b)+Q(\nu) J_{\nu}(k b)}{H_{\nu}^{(2)^{\prime}}(k b)+Q(\nu) H_{\nu}^{(2)}(k b)} H_{\nu}^{(2)}(k \rho)\right] e^{-j \nu \psi} d \nu(14 a)
$$

or

$$
u_{1}=\frac{u_{o}}{2} \int_{-\infty}^{\infty}\left[H_{\nu}^{(1)}(k \rho)-\frac{H_{\nu}^{(1)^{\prime}}(k b)+Q(\nu) H_{\nu}^{(1)}(k b)}{H_{\nu}^{(2)^{\prime}}(k b)+Q(\nu) H_{\nu}^{(2)}(k b)} H_{\nu}^{(2)}(k \rho)\right] e^{-j \nu \psi} d \nu
$$

and

$$
\begin{aligned}
u_{2}= & u_{0} \int_{-\infty}^{\infty}\left[J_{\nu}(k \rho)-\frac{J_{\nu}^{\prime}(k b)+Q(\nu) J_{\nu}(k b)}{H_{\nu}^{(2)}(k b)+Q(\nu) H_{\nu}^{(2)}(k b)} H_{\nu}^{(2)}(k \rho)\right] e^{j \nu \pi / 2} \\
& \cdot \frac{e^{-j \nu(2 \pi+\phi)}+e^{-j \nu(2 \pi-\phi)}}{1-e^{-j 2 \nu \pi}} d \nu
\end{aligned}
$$

in which

$$
\psi=|\phi|-\frac{\pi}{2}
$$


and $Q(\nu)$ is given by (12) upon letting $n \rightarrow \nu$. The field component $u_{1}$, defined above, contains the geometrical optics and dominant surface diffraction contributions whereas $u_{2}$ denotes the creeping waves which circulate around the cylinder more than once. We are interested in an asymptotic evaluation of $u_{1}$ and to do this we must consider separately each of the space regions illustrated in fig. 3.

\section{Field in the Deep Lit and Shadow Re- gions}

In the deep lit region, the geometrical optics incident and reflected rays represent an accurate first-order high-frequency approximation of the total field. The geometrical optics field can be extracted from $u_{1}$ upon performing an asymptotic evaluation of (14) [18] yielding

$$
u^{G O}\left(P_{L}\right) \approx u^{i}\left(P_{L}\right)+u^{i}\left(Q_{R}\right) R_{s, h} \sqrt{\frac{\tilde{\rho}}{\tilde{\rho}+\ell}} e^{-j k \ell}
$$

in which

$$
\begin{aligned}
& R_{s} \approx-\frac{a_{o}^{\prime}-a_{1}^{\prime} \cos \theta^{i}+a_{2}^{\prime} \cos ^{2} \theta^{i}}{a_{o}^{\prime}+a_{1}^{\prime} \cos \theta^{i}+a_{2}^{\prime} \cos ^{2} \theta^{i}} \quad\left(E_{z} \text { - incidence }\right) \\
& R_{h} \approx-\frac{a_{o}-a_{1} \cos \theta^{i}+a_{2} \cos ^{2} \theta^{i}}{a_{o}+a_{1} \cos \theta^{i}+a_{2} \cos ^{2} \theta^{i}} \quad\left(H_{z} \text { - incidence }\right)
\end{aligned}
$$

are the reflection coefficients associated with the coating [9]. The parameter $\theta^{i}$ is illustrated in fig. 4 and $\ell$ is the distance (must be large) from the surface reflection point $Q_{R}$ to the observation point $P_{L}$. Finally, $\tilde{\rho}$ is the reflected ray caustic distance and for a convex cylinder is given by

$$
\tilde{\rho}=\frac{\rho_{g}\left(Q_{R}\right) \cos \theta^{i}}{2}
$$

$\rho_{g}\left(Q_{R}\right)$ is the radius of curvature of the surface at $Q_{R}$ and is equal to $b$ for the circular cylinder. 
For observations in the deep shadow region, a residue series representation of $u_{1}$ is more appropriate. From (14), we obtain

$$
u_{1}=-u_{o} \frac{4}{k b} \sum_{m=1}^{\infty} \frac{H_{\nu_{m}}^{(2)}(k \rho) e^{-j \nu_{m}\left(\phi-\frac{\pi}{2}\right)}}{H_{\nu_{m}}^{(2)}(k b) \frac{\partial}{\partial \nu}\left[H_{\nu}^{(2)}(k b)+Q(\nu) H_{\nu}^{(2)}(k b)\right]_{\nu=\nu_{m}}}
$$

where $\nu_{m}$ are the zeros of the transcendental equation

$$
H_{\nu_{m}}^{(2)^{\prime}}(k b)+Q\left(\nu_{m}\right) H_{\nu_{m}}^{(2)}(k b)=0
$$

Obviously (20) does not provide a ray-picture interpretation of the creepingwave diffraction which is desirable for generalizations to non-circular cylinders. To recast (20) in a form compatible with the Keller type GTD format, the Debye approximation must be employed for the Hankel function for $k \rho \gg\left|\nu_{m}\right|$. This yields

$$
u_{1}^{d}\left(P_{s}\right) \approx u^{i}\left(Q_{1}\right) T_{s, h} \frac{e^{-j k s}}{\sqrt{s}}
$$

where

$$
T_{s, h}=-\sum_{m=1}^{\infty} \mathcal{D}_{m}\left(Q_{1}\right) \cdot e^{-j \nu_{m} \theta} \cdot \mathcal{D}_{m}\left(Q_{2}\right)
$$

is the corresponding diffraction coefficient and for the circular cylinder

$$
\begin{aligned}
\mathcal{D}_{m}\left(Q_{1}\right) & =\mathcal{D}_{m}\left(Q_{2}\right) \\
& =\left[\sqrt{\frac{2}{\pi}} \frac{4}{k^{2} b} \frac{e^{j \frac{\pi}{4}}}{H_{\nu_{m}}^{(2)}(k b) \frac{\partial}{\partial \nu}\left\{H_{\nu}^{(2)}(k b)+Q(\nu) H_{\nu}^{(2)}(k b)\right\}_{\nu=\nu_{m}}}\right]^{\frac{1}{2}}
\end{aligned}
$$

with $\theta$ as defined in figure 5 .

A generalization of (22) - (24) for convex cylinders is now possible by making the replacements

$$
\begin{aligned}
b & \rightarrow \rho_{g}\left(Q_{1,2}\right) \\
e^{-j \nu_{m} \theta} & \rightarrow \exp \left[-j \int_{t^{\prime}\left(Q_{1}\right)}^{t^{\prime}\left(Q_{2}\right)} \frac{\nu_{m}\left(t^{\prime}\right)}{\rho_{g}\left(t^{\prime}\right)} d t^{\prime}\right]
\end{aligned}
$$

Consequently, the attachment coefficient $\mathcal{D}_{m}\left(Q_{1}\right)$ is no longer equal to the launching coefficient $\mathcal{D}_{m}\left(Q_{2}\right)$. 


\section{Field in the Transition Region}

The geometrical optics and creeping wave solutions presented in the previous section are not valid in the transition region close to the shadow boundaries as illustrated in figure 3. New uniform expressions are, therefore, required to overcome this limitation. These can be derived following the procedure adapted by Pathak [5] for a perfectly conducting convex cylinder. For the lit region we find

$$
u_{1}\left(P_{L}\right) \approx u^{i}\left(P_{L}\right)+u^{i}\left(Q_{R}\right) R_{s, h} \sqrt{\frac{\tilde{\rho}}{\tilde{\rho}+\ell}} e^{-j k \ell}
$$

where $R_{s, h}$ is now given by

$$
\begin{gathered}
R_{s, h}=-\sqrt{\frac{-4}{x^{\prime}}} \exp \left\{-j \frac{\left(x^{\prime}\right)^{3}}{12}\right\}\left[\frac{e^{-j \frac{\pi}{4}}}{2 x^{\prime} \sqrt{\pi}}\left\{1-F\left(2 k \ell \cos ^{2} \theta^{i}\right)\right\}+G\left(x^{\prime}, q\right)\right] \\
F(x)=2 j \sqrt{x} e^{-j x} \int_{x}^{\infty} e^{-j x^{2}} d x \\
x^{\prime}=-2 m\left(Q_{R}\right) \cos \theta^{i} \\
m\left(Q_{R}\right)=\left\{\frac{k \rho_{g}\left(Q_{R}\right)}{2}\right\}^{\frac{1}{3}} \\
\tilde{\rho}=\frac{\rho_{g}\left(Q_{R}\right) \cos \theta^{i}}{2}
\end{gathered}
$$

$F(x)$ is the UTD transition function [19] and $G\left(x^{\prime}, q\right)$ is defined by

$$
\begin{aligned}
G\left(x^{\prime}, q\right) & =\frac{e^{-j \frac{\pi}{\tau}}}{\sqrt{\pi}} \int_{-\infty}^{\infty} \frac{V^{\prime}(\tau)-q(\tau) V(\tau)}{W_{2}^{\prime}(\tau)-q(\tau) W_{2}(\tau)} e^{-j x \tau} d \tau \\
g(\tau) & =m Q(\nu)
\end{aligned}
$$


where $V(\tau)$ and $W_{1,2}(\tau)$ are the Fock-type Airy functions [15]

$$
\begin{aligned}
& 2 j V(\tau)=W_{1}(\tau)-W_{2}(\tau) \\
& W_{1,2}(\tau)=\frac{1}{\sqrt{\pi}} \int_{\Gamma_{1,2}} e^{\tau t-t^{3} / 3} d t
\end{aligned}
$$

The contour $\Gamma_{1}$ runs from $\infty e^{-j \frac{2 \pi}{3}}$ to $\infty-j \epsilon$ and $\Gamma_{2}$ is the complex conjugate of $\Gamma_{1}$. We remark that for the case of SIBC $q(\tau)$ will be replaced by a constant term $q$.

For the shadow region, we have

$$
u_{1}\left(P_{s}\right) \approx u^{i}\left(Q_{1}\right) T_{s, h} \frac{e^{-j k s}}{\sqrt{s}}
$$

where the diffraction coefficient $T_{s, h}$ is now redefined as

$$
T_{s, h}=-\sqrt{m\left(Q_{1}\right) m\left(Q_{2}\right)} \sqrt{\frac{2}{k}} e^{-j k t}\left[\frac{e^{-j \frac{\pi}{4}}}{2 x \sqrt{\pi}}\{1-F(k s \tilde{a})\}+G(x, q)\right]
$$

in which

$$
\begin{aligned}
x & =\int_{t^{\prime}\left(Q_{1}\right)}^{t^{\prime}\left(Q_{2}\right)} \frac{m\left(t^{\prime}\right)}{\rho_{g}\left(t^{\prime}\right)} d t^{\prime} \\
t & =\int_{t^{\prime}\left(Q_{1}\right)}^{t^{\prime}\left(Q_{2}\right)} d t^{\prime} \\
\tilde{a} & =\frac{x^{2}}{2 m\left(Q_{1}\right) m\left(Q_{2}\right)}
\end{aligned}
$$

As is usually the case, (27)-(40) were first derived for the circular cylinder and were subsequently generalized for non-circular convex cylinders.

\section{Field in the Close Vicinity of the Conex Cylinder}

In all of the above derivations we have assumed that $\ell$ is large. Consequently, the given expressions are not adequate in describing the field in 
the close vicinity of the cylinder. In this case, it is possible to obtain a suitable approximation of the integral (14) by replacing the Hankel and Bessel functions in terms of Airy integrals and then emplyoing Taylor series expansions for the Airy integrals.

Following a procedure similar to the one given in [5], the resulting expressions for an arbitrary smooth convex cylinder are

$$
u_{1}(P) \approx u^{i}\left(P_{N}\right)\left[e^{-j h z^{\prime}}-\sum_{n=0}^{5} \frac{(-1)^{n}}{n !}\left(j h z^{\prime}\right)^{n}+e^{\frac{-j\left(z^{\prime}\right)^{3}}{3}}\left\{\Lambda_{1}\left(z^{\prime}\right)-\Lambda_{2}\left(z^{\prime}\right)\right\}\right]
$$

when $P_{N}$ is in the lit region, and

$$
u_{1}(P) \approx u^{i}\left(Q_{1}\right) e^{-j k t}\left[\frac{\rho_{g}\left(P_{N}\right)}{\rho_{g}\left(Q_{1}\right)}\right]^{\frac{-1}{6}}\left\{\Lambda_{1}(z)-\Lambda_{2}(z)\right\}
$$

when $P_{N}$ is in the shadow region. In (42) and (43)

$$
\begin{gathered}
\Lambda_{1}(D)=g_{1}(D)+j \frac{h^{2}}{2 !} g_{1}^{\prime}(D)-\frac{h^{3}}{3 !} g_{1}(D)-\frac{h^{4}}{4 !} g_{1}^{\prime \prime}(D)-j 4 \frac{h^{5}}{5 !} g_{1}^{\prime}(D) \\
\Lambda_{2}(D)=h g_{2}(D)+j \frac{h^{3}}{3 !} g_{2}^{\prime}(D)-2 \frac{h^{4}}{4 !} g_{2}(D)-\frac{h^{5}}{5 !} g_{2}^{\prime \prime}(D) \\
g_{1}(D)=\frac{1}{\sqrt{\pi}} \int_{-\infty}^{\infty} \frac{e^{-j D \tau}}{W_{2}^{\prime}(\tau)-q(\tau) W_{2}(\tau)} d \tau \\
g_{2}(D)=\frac{1}{\sqrt{\pi}} \int_{-\infty}^{\infty} \frac{q(\tau) e^{-j D \tau}}{W_{2}^{\prime}(\tau)-q(\tau) W_{2}(\tau)} d \tau \\
z^{\prime}=-m\left(P_{N}\right) \cos \theta^{i}
\end{gathered}
$$

for $P_{N}$ in the lit region and

$$
z=\int_{t^{\prime}\left(Q_{1}\right)}^{t^{\prime}\left(P_{N}\right)} \frac{m\left(t^{\prime}\right)}{\rho_{g}\left(t^{\prime}\right)} d t^{\prime}
$$


for $P_{N}$ in the shadow region

$$
\begin{aligned}
t & =\int_{t^{\prime}\left(Q_{1}\right)}^{t^{\prime}\left(P_{N}\right)} d t^{\prime} \\
h & =\frac{k d\left(P_{N}\right)}{m\left(P_{N}\right)} \\
d\left(P_{N}\right) & =\rho-\rho_{g}\left(P_{N}\right)
\end{aligned}
$$

The points $P$ and $P_{N}$ in the lit and shadow regions are illustrated in figure 6. We remark that when the cylinder's surface obeys the SIBC, $g_{1,2}(D)$ simplify to

$$
\begin{gathered}
g_{1}(D) \rightarrow g(D)=\frac{1}{\sqrt{\pi}} \int_{-\infty}^{\infty} \frac{e^{-j D \tau}}{W_{2}^{\prime}(\tau)-q W_{2}(\tau)} d \tau \\
g_{2}(D) \rightarrow m Q g(D)
\end{gathered}
$$

It should be noted that (42) which is applicable in the lit region may not be of sufficient accuracy in the case of slowly attenuating creeping and/or surface waves. An improved result can then be obtained by adding (42) and (43) with $t>\pi b$ (in case of a circular cylinder of radius b). Clearly, the addition of (43) corresponds to the contribution of the creeping wave that has travelled the minimum distance on the cylinder's surface to reach $P_{N}$. The contribution of those creeping waves that travelled more than once around the cylinder is given by $u_{2}$ and could be added to $u_{1}$ if greater accuracy is required.

The functions $g_{1}(D), g_{2}(D)$ and $G(x, q)$ are Fock-type integrals that are formally the Fourier transform of a slowly varying factor consisting of a quotient of terms containing Airy functions and their derivatives. A computationally efficient scheme was proposed by Pearson [16] for the evaluation of these integrals. The scheme is an extention of the Fourier trapezoidal rule, devised by Tuck [17], to treat the rotated-ray exponential behavior occuring in the integrals. The sampling frequency used in the computation is dictated by the slowly varying Airy-function factor in the integrands. Sufficiently accurate results have been obtained for both lossy and non-lossy coatings on a perfectly conducting cylinder using this scheme. 


\section{Numerical Results}

The UTD expressions derived in the previous sections provide a complete set of equations for the computation of the total field in any region of interest. In this section, we present some curves which validate the accuracy of the derived expressions by comparison with data based on the moment method and eigenfunction solutions.

In figure 8 the eigenfunction solutions based on GIBC and SIBC are compared with the exact one for a coated cylinder which demonstrates an improved simulation (with respect to the standard impedance boundary conditions - SIBC). To show the validity of the UTD solution in the case of a convex cylinder, a special case of an elliptical cylinder (see fig. 7) is considered in figure 9. Data based on the moment method solution are compared with the one obtained from the UTD solution in conjunction with low (equ. 3,4) and high contrast (equ. 5,6) boundary conditions. Figure 10 verifies the asymptotic solution, developed for the field point in the close vicinity of a convex cylinder. We remark that the approximations used for the Hankel functions for the derivation of (42) and (43) become less accurate for some values of $\epsilon_{r}$ and $\mu_{r}$ in case of non-lossy coatings. This can be avoided by using more accurate approximations for the Hankel functions. Finally, figure 11 demonstrates the use of GIBC to simulate multilayer coatings by simply redefining the material constants $a_{m}$ and $a_{m}^{\prime}$ as discussed in $[10,13]$.

A difficulty in implementing the expressions derived in this paper was the evaluation of the Fock-type integrals $G(x, q), g_{1}(D)$ and $g_{2}(D)$ as well as the determination of the zeros corresponding to (21). The Fock-type integrals were evaluated by employing the method described in [16] and the zeros of (21) were determined using the routine given in [20].

\section{Acknowledgements}

The authors are indebted to Dr. L. Wilson Pearson for providing the subroutines for the computation of the Fock-type integrals and to Dr. N. Wang for the routine to compute complex order Hankel functions. The complex zero search routine was provided by Mr. Stanley Locus. 


\section{Appendix}

Consider an elliptical cylinder as an example of a convex geometry shown in figure 7. The geometrical and field parameters associated with this surface are listed below:

Incident field

At the observation point $(\rho, \phi)$ a unit amplitude plane wave incident at an angle $\phi_{i}$ is specified by (phase of all fields will be referenced to the origin)

$$
u^{i}(\rho, \phi)=e^{j k \rho \cos \left(\phi-\phi_{i}\right)}
$$

Geometry

The surface of an elliptical cylinder is described by a parameterized vector as

$$
\mathbf{r}(\gamma)=a \cos \gamma \hat{x}+b \sin \gamma \hat{y}
$$

where the parameter $\gamma(0 \leq \gamma \leq 2 \pi)$ is related to the angle $\phi$ via the relation

$$
\tan \phi=\frac{b}{a} \tan \gamma
$$

Also, $a$ and $b$ are the half lengths of the major and minor axis of the elliptical cylinder, respectively. The radius of curvature at any point $\gamma$ on the elliptical surface is

$$
\rho_{g}(\gamma)=\frac{\left(a^{2} \sin ^{2} \gamma+b^{2} \cos ^{2} \gamma\right)^{3 / 2}}{a b}
$$

and the unit tangent and normal to the surface are given by

$$
\begin{aligned}
& \hat{t}=\frac{\mathbf{r}^{\prime}(\gamma)}{\left|\mathbf{r}^{\prime}(\gamma)\right|}=\frac{-a \sin \gamma \hat{x}+b \cos \gamma \hat{y}}{\sqrt{a^{2} \sin ^{2} \gamma+b^{2} \cos ^{2} \gamma}} \\
& \hat{n}=\hat{t} \times \hat{z}=\frac{b \cos \gamma \hat{x}+a \sin \gamma \hat{y}}{\sqrt{a^{2} \sin ^{2} \gamma+b^{2} \cos ^{2} \gamma}}
\end{aligned}
$$

Location of the reflection point $Q_{R}$ 
The direction of the incident ray at the point of reflection $Q_{R}$ is

$$
\hat{s}^{i}=-\cos \phi_{i} \hat{x}-\sin \phi_{i} \hat{y}
$$

Let $Q_{R}$ be the reflection point corresponding to $\gamma=\gamma_{R}$. The direction of the reflected ray is then given by

$$
\hat{\boldsymbol{s}}^{r}=\frac{\left(\rho \cos \phi-a \cos \gamma_{R}\right) \hat{x}+\left(\rho \sin \phi-b \sin \gamma_{R}\right) \hat{y}}{\sqrt{\left(\rho \cos \phi-a \cos \gamma_{R}\right)^{2}+\left(\rho \sin \phi-b \sin \gamma_{R}\right)^{2}}}
$$

Enforcement of the reflection law $\hat{n} \cdot \hat{s}^{i}=-\hat{n} \cdot \hat{s}^{r}$ at $Q_{R}$ then yields the transcendental equation

$$
\begin{aligned}
& \frac{\rho\left(b \cos \phi \cos \gamma_{R}+a \sin \phi \sin \gamma_{R}\right)-a b}{\sqrt{\rho^{2}+a^{2} \cos ^{2} \gamma_{R}+b^{2} \sin \gamma_{R}-2 \rho\left(a \cos \phi \cos \gamma_{R}+b \sin \phi \sin \gamma_{R}\right)}} \\
& =b \cos \phi_{i} \cos \gamma_{R}+a \sin \phi_{i} \sin \gamma_{R}
\end{aligned}
$$

to be solved for $\gamma_{R}$ for each observation angle $\phi$. The solution must of course be done numerically using an iterative routine such as the NewtonRaphson's method. Given $\gamma_{R}$ the incident field at $Q_{R}$

$$
u^{i}\left(Q_{R}\right)=e^{j k \ell_{R}}
$$

where

$$
\ell_{R}=\sqrt{a^{2} \cos ^{2} \gamma_{R}+b^{2} \sin ^{2} \gamma_{R}} \cos \left(\phi_{\mathrm{r}}-\phi_{i}\right)
$$

with

$$
\phi_{r}=\tan ^{-1}\left\{\frac{b}{a} \tan \gamma_{R}\right\}
$$

The reflected ray caustic distance at $Q_{R}$ is given by

$$
\tilde{\rho}=\frac{\rho_{g}\left(Q_{R}\right) \cos \theta^{i}}{2}
$$


where

$$
\begin{aligned}
\rho_{g}\left(Q_{R}\right) & =\frac{\left(a^{2} \sin ^{2} \gamma_{R}+b^{2} \cos ^{2} \gamma_{R}\right)^{3 / 2}}{a b} \\
\cos \theta^{i} & =\hat{n}\left(Q_{R}\right) \cdot \hat{s}^{r}=\frac{b \cos \phi_{i} \cos \gamma_{R}+a \sin \phi_{i} \sin \gamma_{R}}{\sqrt{a^{2} \sin ^{2} \gamma_{R}+b^{2} \cos ^{2} \gamma_{R}}}
\end{aligned}
$$

with $\ell$ in (17), (27) and (28) found from

$$
\ell=\sqrt{\left(\rho \cos \phi-a \cos \gamma_{R}\right)^{2}+\left(\rho \sin \phi-b \sin \gamma_{R}\right)^{2}}
$$

Attachment and detachment points for the surface diffracted ray

The attachment point $Q_{1}$ on the surface of the cylinder shown in figure 7 can be determined by enforcing the generalized Fermat principle implying that at $Q_{1}\left(a \cos \gamma_{1}, b \sin \gamma_{1}\right)$

$$
\hat{n}\left(Q_{1}\right) \cdot \hat{s}^{i}=0
$$

this yields

$$
\gamma_{1}=\tan ^{-1}\left\{-\frac{b}{a} \cot \phi_{i}\right\}
$$

Similarly, the surface detachment point $Q_{2}\left(a \cos \gamma_{2}, b \sin \gamma_{2}\right)$ is determined by enforcing

$$
\hat{n}\left(Q_{2}\right) \cdot \hat{s}=0
$$

where

$$
\hat{s}=\frac{\left(\rho \cos \phi-a \cos \gamma_{2}\right) \hat{x}+\left(\rho \sin \phi-b \sin \gamma_{2}\right) \hat{y}}{\sqrt{\left(\rho \cos \phi-a \cos \gamma_{2}\right)^{2}+\left(\rho \sin \phi-b \sin \gamma_{2}\right)^{2}}}
$$

This leads to the transcendental equation

$$
\rho\left(a \sin \phi \sin \gamma_{2}+b \cos \phi \cos \gamma_{2}\right)-a b=0
$$

which can be solved numerically for $\gamma_{2}$.

Incident field at $Q_{1}$ is 


$$
u^{i}\left(Q_{1}\right)=e^{j k r_{1} \cos \left(\phi_{1}-\phi_{i}\right)}
$$

where

$$
\begin{aligned}
& r_{1}=\sqrt{a^{2} \cos ^{2} \gamma_{1}+b^{2} \sin ^{2} \gamma_{1}} \\
& \phi_{1}=\tan ^{-1}\left\{\frac{b}{a} \tan \gamma_{1}\right\}
\end{aligned}
$$

Also, the parameters $x$ and $t$ in (38) and (39) are, respectively, given by

$$
\begin{aligned}
& x=\left\{\frac{k a^{2} b^{2}}{2}\right\}^{1 / 3} \int_{\gamma_{1}}^{\gamma_{2}} \frac{d \gamma}{\sqrt{a^{2} \sin ^{2} \gamma+b^{2} \cos ^{2} \gamma}} \\
& t=\int_{\gamma_{1}}^{2} \sqrt{a^{2} \sin ^{2} \gamma+b^{2} \cos ^{2} \gamma d \gamma}
\end{aligned}
$$

The integrals in (A.25) and (A.26) must be computed numerically.

\section{References}

[1] N. Wang, "Regge poles, natural frequencies, and surface wave resonance of a circular cylinder with a constant surface impedance," IEEE Trans. Antenna \& Propagat., Vol. AP-30, Nov. 1982.

[2] - - - "Electromagnetic scattering from a dielectric coated circular cylinder," IEEE Trans. Antenna \& Propagat., Vol. AP-33, Sept. 1985.

[3] J. R. Wait and A. M. Conda, "Diffraction of electromagnetic waves by smooth obstacles for grazing angles," J. Res. Bur. Stand., Vol. 63D, pp. 181-197, 1959.

[4] - - -, "Pattern of an antenna on a curved lossy surface," IRE Trans. Antennas 8 Propagat., Vol. AP-6, pp. 348-359, Oct. 1958.

[5] P. H. Pathak, "An asymptotic Analysis of the scattering of plane waves by a smooth convex cylinder," Radio Sci., Vol. 14, pp. 419-435, 1979. 
[6] J. B. Keller, "Diffraction by a convex cylinder," IEEE Trans. Antenna $\&$ Propagat., Vol. AP-24, pp. 312-321, 1956.

[7] - - -, "Geometrical theory of diffraction," J. Opt. Soc. Amer., Vol. 52, pp. 116-130, 1962.

[8] H. Kim and N. Wang, "UTD solution for electromagnetic scattering by a circular cylinder with thin lossy coatings," IEEE Trans. Antenne \& Propagat., Vol. AP-37, pp. 1463-1472, Nov. 1989.

[9] T. B. A. Senior and J. L. Volakis, "Derivation and application of a class of generalized boundary conditions," IEEE Trans. Antenna 8 Propagat., Vol. AP-37, pp. 1566-1572, Dec. 1989.

[10] M. A. Ricoy and J. L. Volakis, "Derivation of generalized transition/boundary conditions for planar multiple layer structures," Radio Sci., Vol. 25, pp. 391-405, July-Aug. 1990.

[11] - - - "Diffraction by a symmetric material junction, Part I: general solution," submitted for publication.

[12] - - -, "Diffraction by a symmetric material junction, Part II: boundary conditions," submitted for publication.

[13] J. L. Volakis and H. H. Syed, "Application of higher order boundary conditions to scattering by multilayered coated cylinders," $J$. of Electromagnetic Waves and Applications. (in press)

[14] J. J. Bowman, T. B. A. Senior and P. L. E. Uslenghi, "Electromagnetic and Acoustic Scattering by Simple Shapes," Amsterdam: North-Holland, 1969.

[15] N. A. Logan and K. S. Yee, "Electromagnetic Waves," edited by R. E. Langer, University of Wisconsin Press, Madison.

[16] L. W. Pearson, "A scheme for automatic computation of Fock-type integrals," IEEE Trans. Antenna \& Propagat., Vol. AP-35, pp. 11111118 , Oct. 1987. 
[17] O. A. Tuck, "A simple 'Finlon-trapzoid' rule," Math. Comp., Vol. 21, pp. 239-241, 1967.

[18] L. Felson and N. Marcuvitz, "Radiation and Scattering of Waves," Prentice Hall, 1973.

[19] R. G. Kouyoumjian and P. H. Pathak, "A uniform geometrical theory of diffraction for an edge in a perfectly conducting surface," Proc. $I E E E$, Vol. 62, pp. 1448-1461, 1974.

[20] B. K. Singaraju, D. V. Giri and C. E. Baum, "Further development in the application of contour integration to the evaluation of the zero of analytical functions and relevent computer programs," Math. Note 42, March 1976. 


\section{List of Figures}

Fig. 1. Ilustration of a three-layer coated cylinder.

Fig. 2. (a) Coated circular cylinder (b) equivalent cylinder with coating simulated with generalized impedance boundary conditions.

Fig. 3. Different regions associated with the plane wave scattering from a smooth convex cylinder.

Fig. 4. Reflected ray path for the lit region.

Fig. 5. Ray paths for the shadow region.

Fig. 6. The projection of the field point $P$ in the direction of the surface normal at $P_{N}$.

Fig. 7. Elliptical cylinder configuration.

Fig. 8. Bistatic scattering pattern of a circular cylinder with $b=3 \lambda$, Hpolarization (a) $\epsilon_{r}=4, \mu_{r}=1, \delta=0.07 \lambda, \rho=5 \lambda$ (b) $\epsilon_{r}=8$, $\mu_{r}=1, \delta=0.2 \lambda, \rho=3.05 \lambda$.

Fig. 9. Bistatic scattering pattern of an elliptical cylinder with $a=2 \lambda$, $b=1 \lambda, \rho=5 \lambda, \phi_{i}=0$ (a) E-polarization, $\epsilon_{r}=4, \mu_{r}=1$, $\delta=0.07 \lambda(\mathrm{b}) \mathrm{H}$-polarization, $\epsilon_{r}=8, \mu_{r}=1, \delta=0.2 \lambda$.

Fig. 10. Bistatic scattering pattern of a circular cylinder with $b=3 \lambda$, $\rho=3.05 \lambda, \phi_{i}=0$ (a) E-polarization, $\epsilon_{r}=4, \mu_{r}=1, \delta=0.07 \lambda$ (b) H-polarization, $\epsilon_{r}=8, \mu_{r}=1, \delta=0.2 \lambda$.

Fig. 11. Bistatic scattering pattern of a three-layer coated circular cylinder with $b=3 \lambda, \phi_{i}=0, \epsilon_{r 1}=3-j 0.1, \epsilon_{r 2}=4-j 0.3, \epsilon_{r 3}=7-j 1.5$, $\mu_{r 1}=\mu_{r 2}=\mu_{r 3}=1, \delta_{1}=0.01 \lambda, \delta_{2}=0.02 \lambda, \delta_{3}=0.03 \lambda$ (a) H-polarization, $\rho=5 \lambda$ (b) E- polarization, $\rho=3.05 \lambda$. 


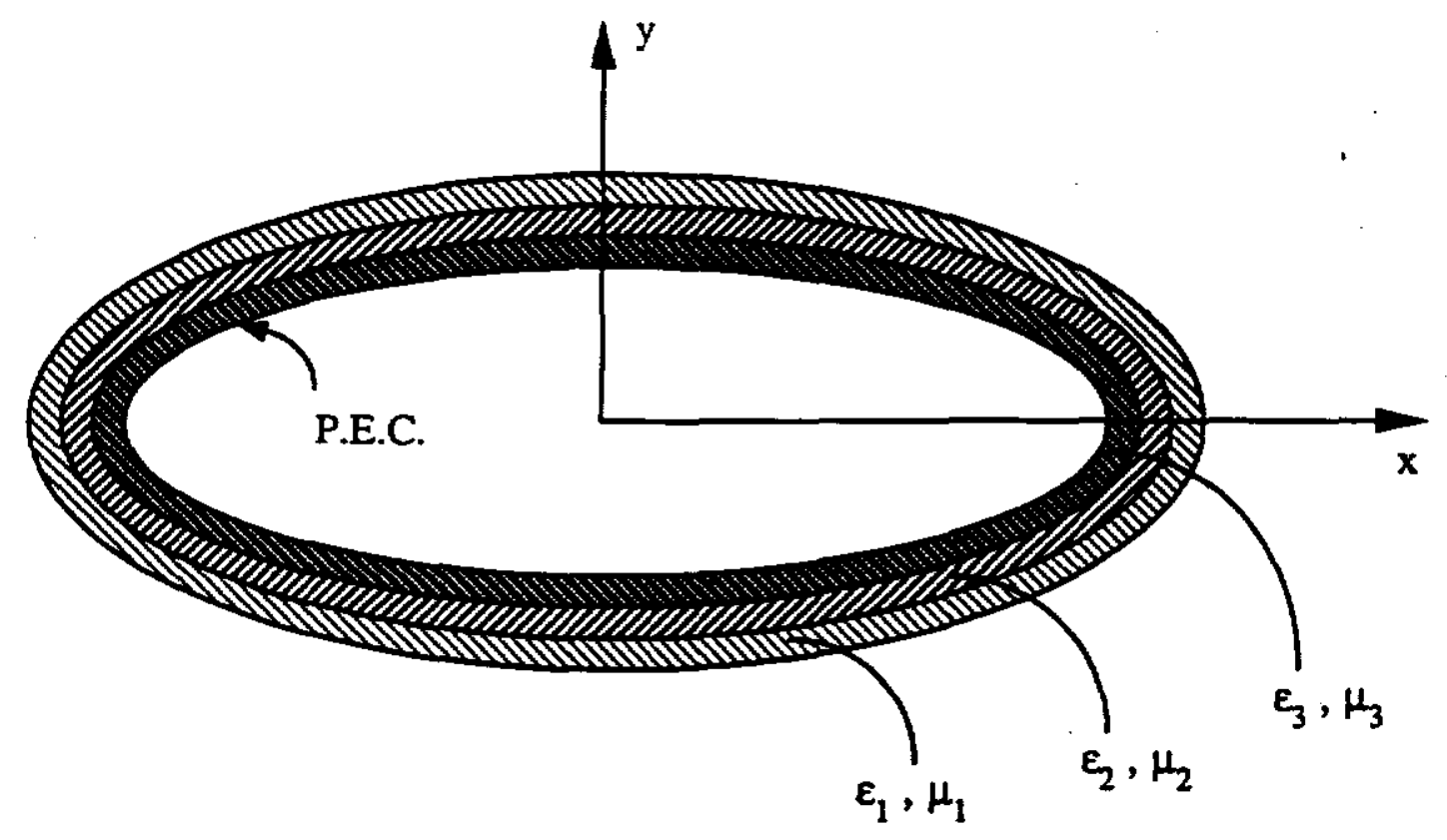

Fig.1. Illustration of a three-layer coated cylinder. 


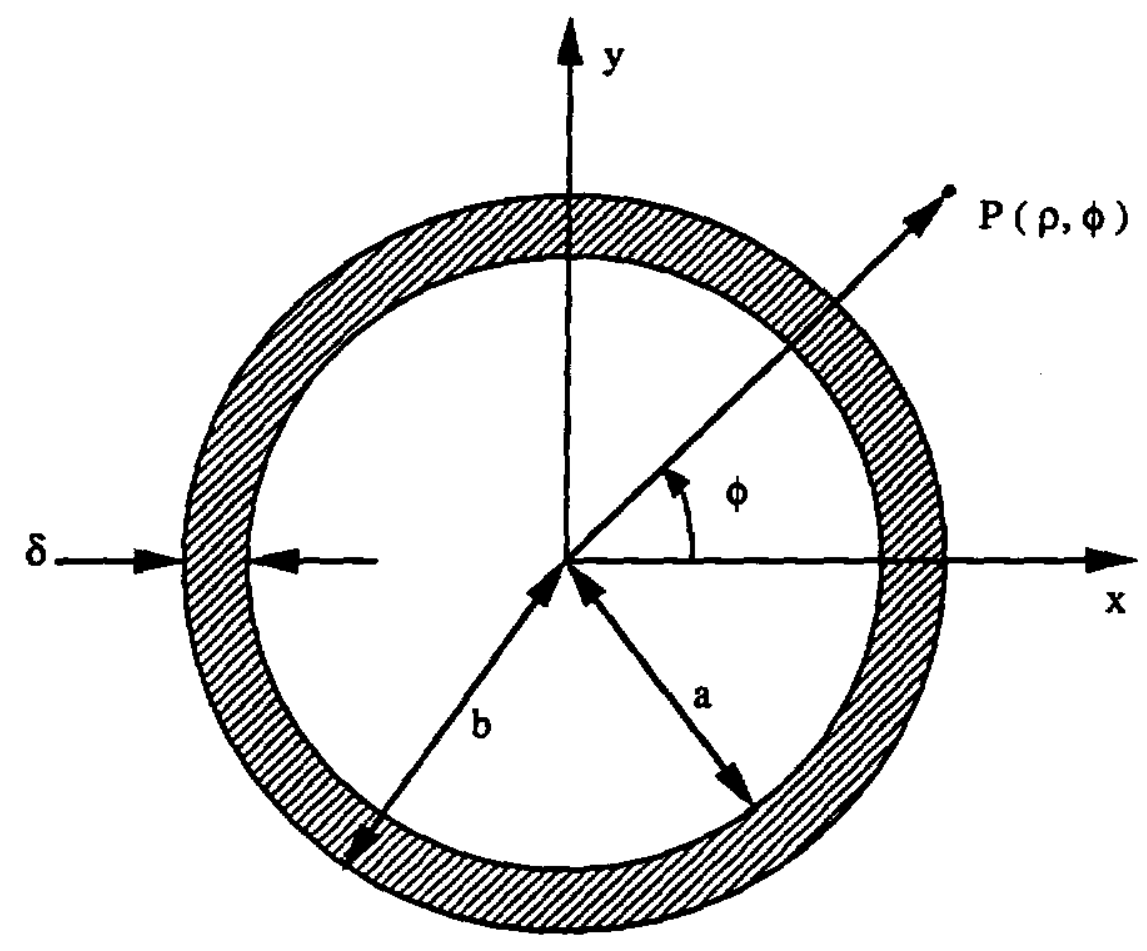

(a)

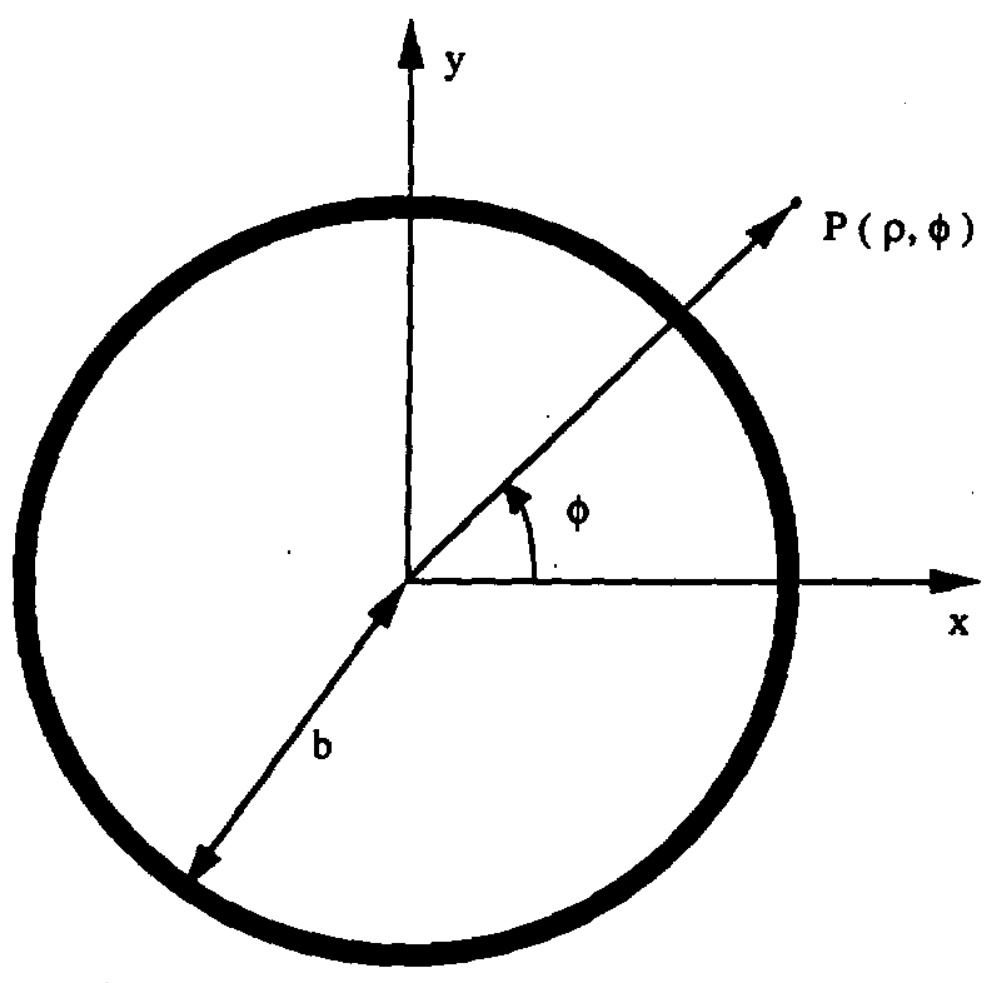

(b)

Fig.2. (a) Coated circular cylinder (b) equivalent cylinder with coating simulated with generalized impedance boundary conditions. 


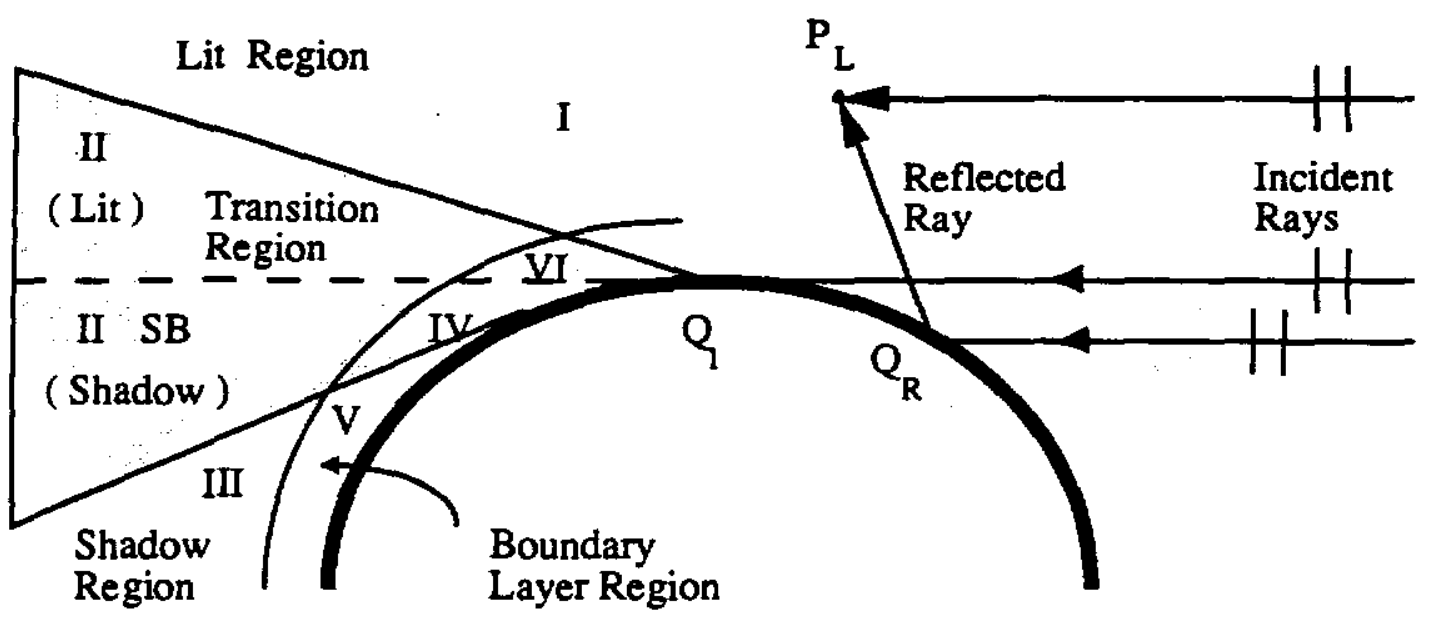

Fig. 3. Different regions associated with the plane wave scattering from a smooth convex cylinder.

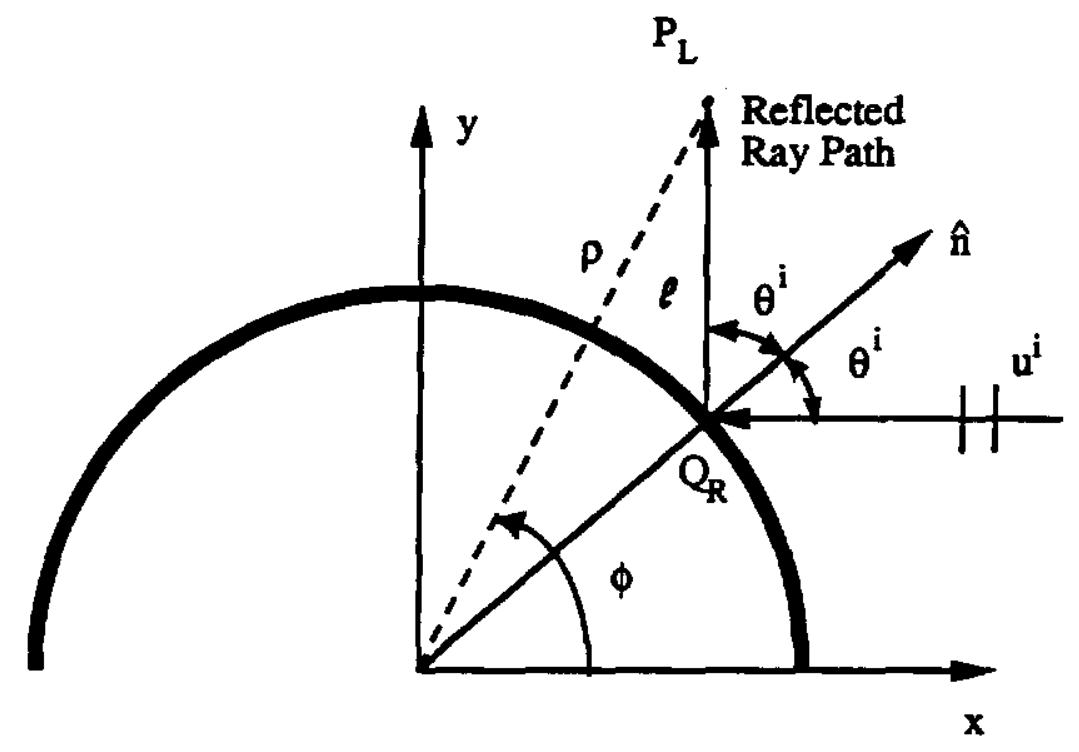

Fig. 4. Reflected ray path for the lit region. 


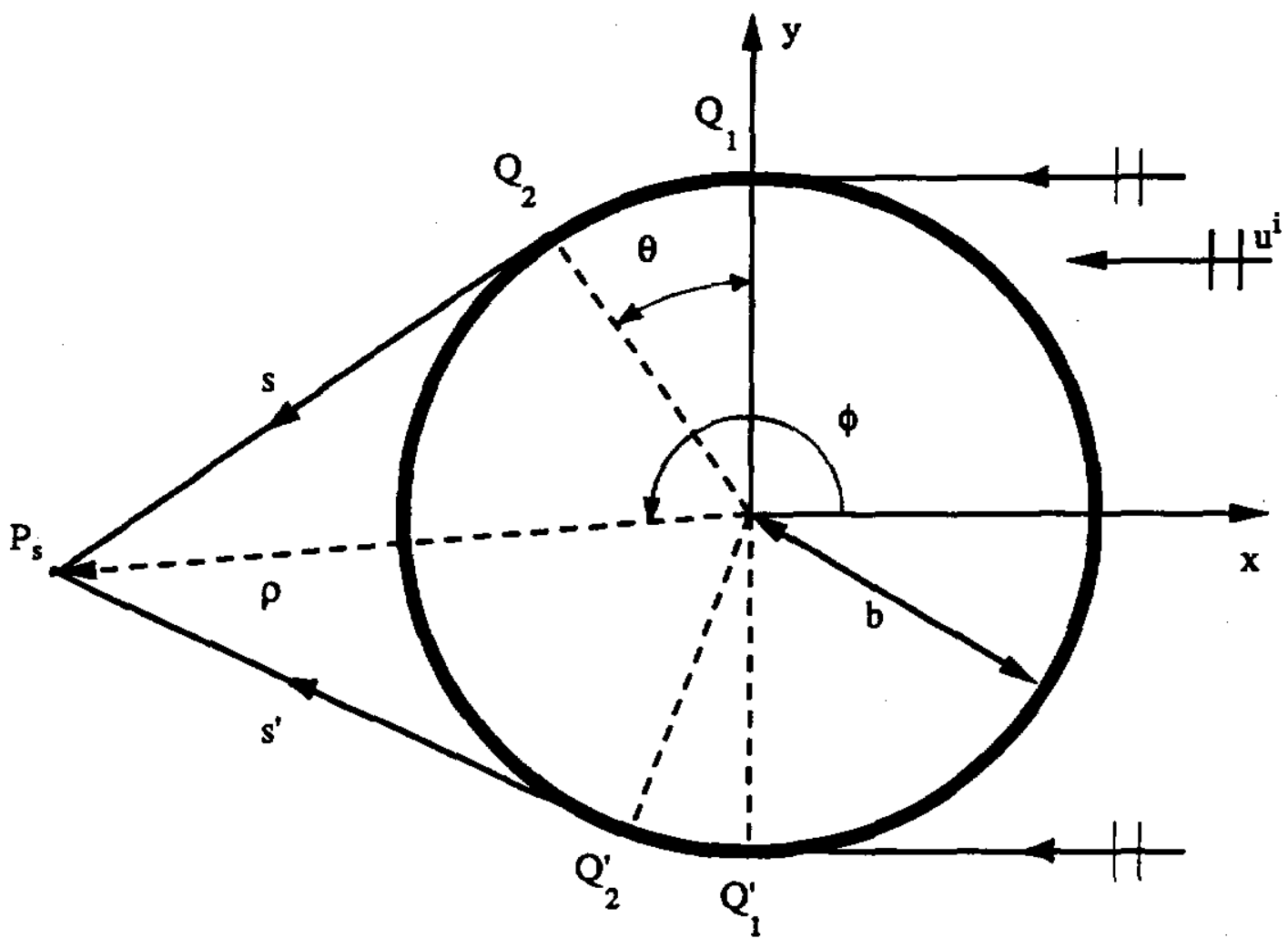

Fig. 5. Ray paths for the shadow region.

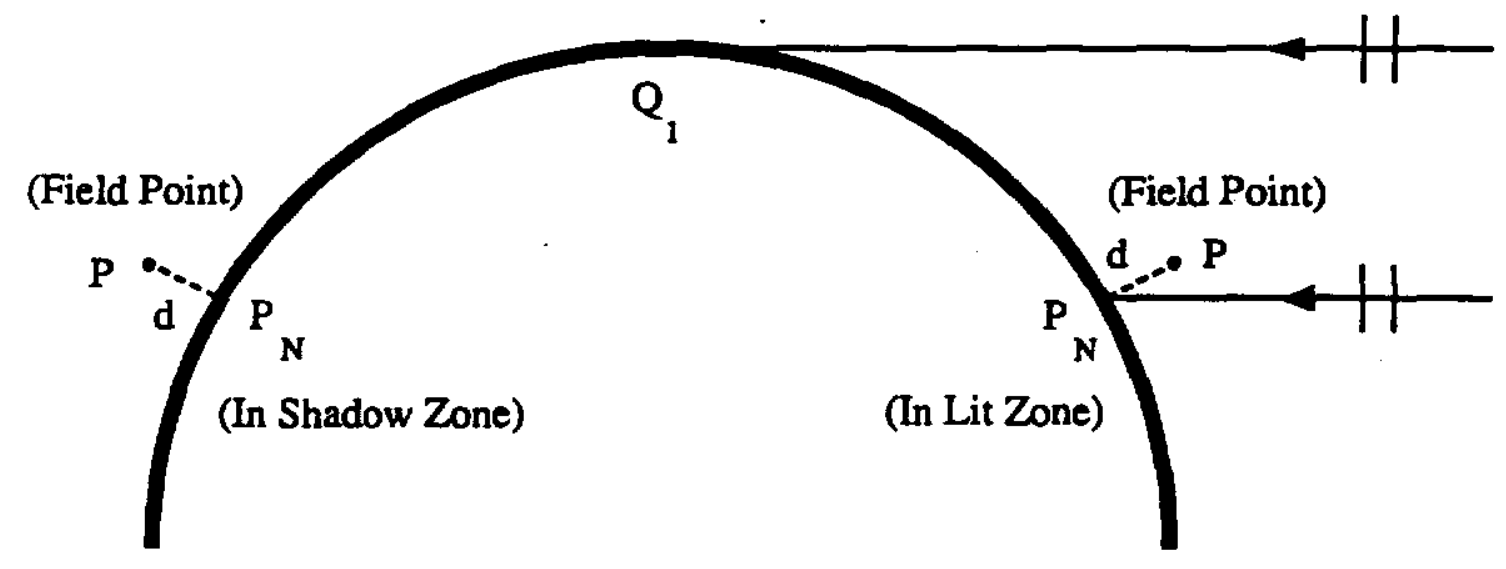

Fig. 6. The projection of the field point $P$ in the direction of the surface normal at $P_{N}$. 


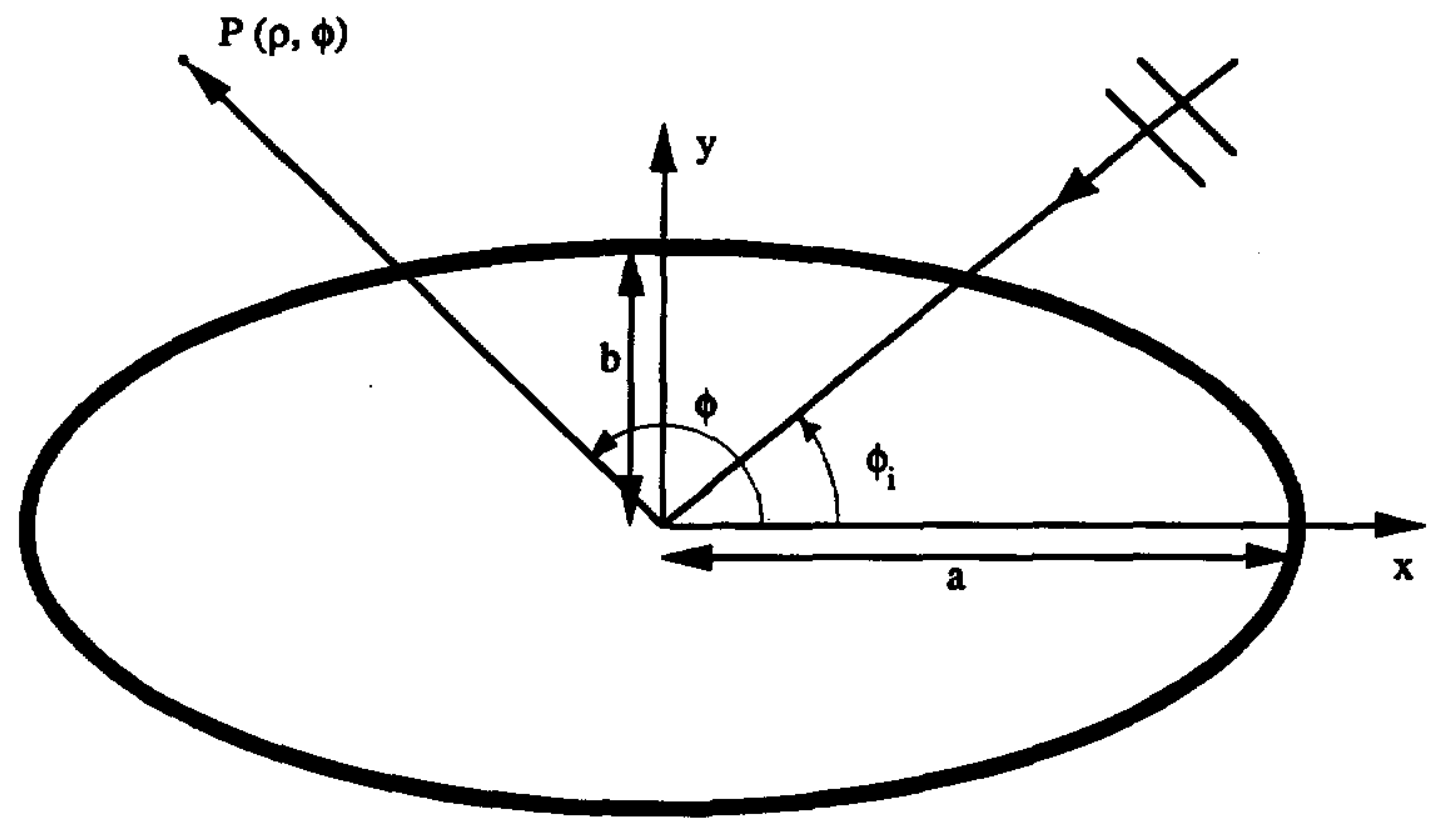

Fig. 7. Elliptical cylinder configuration. 


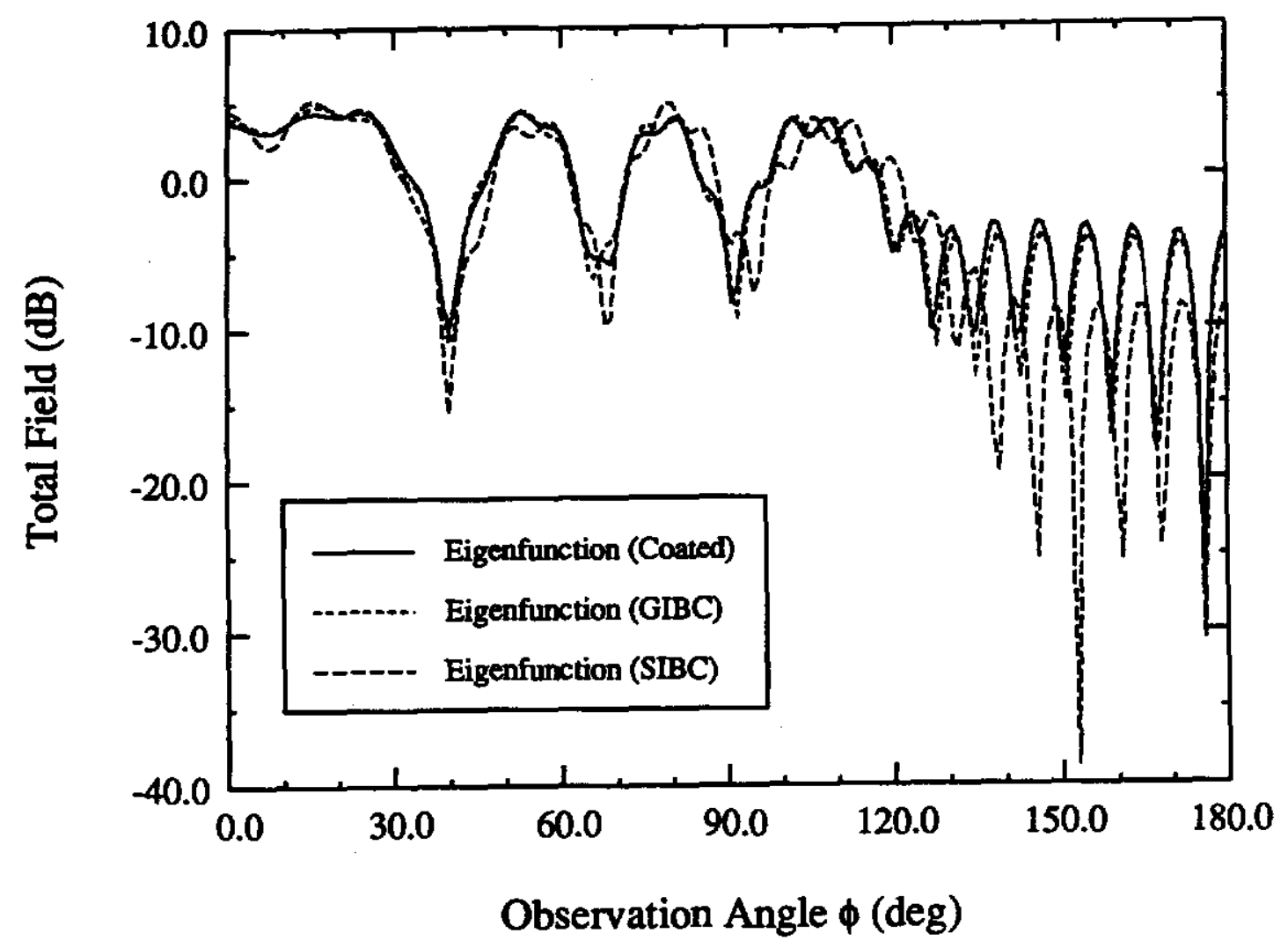

(a)

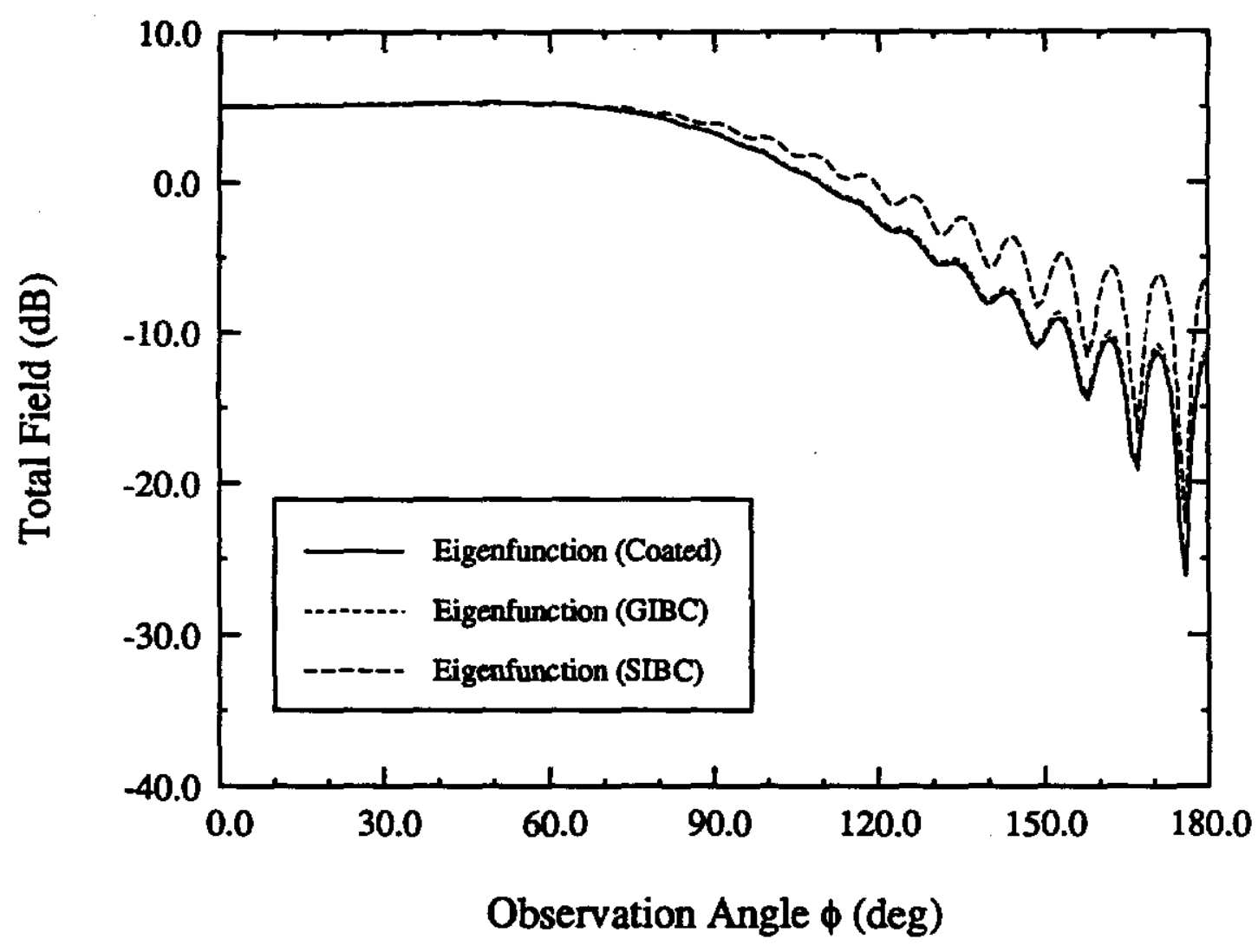

(b)

Fig. 8. Bistatic scattering pattern of a circular cylinder with $b=3 \lambda$, H-polarization (a) $\varepsilon_{\mathrm{T}}=4$, $\mu_{\mathrm{T}}=1, \delta=0.07 \lambda, \rho=5 \lambda$ (b) $\varepsilon_{\mathrm{T}}=8, \mu_{\mathrm{T}}=1, \delta=0.2 \lambda, \rho=3.05 \lambda$. 


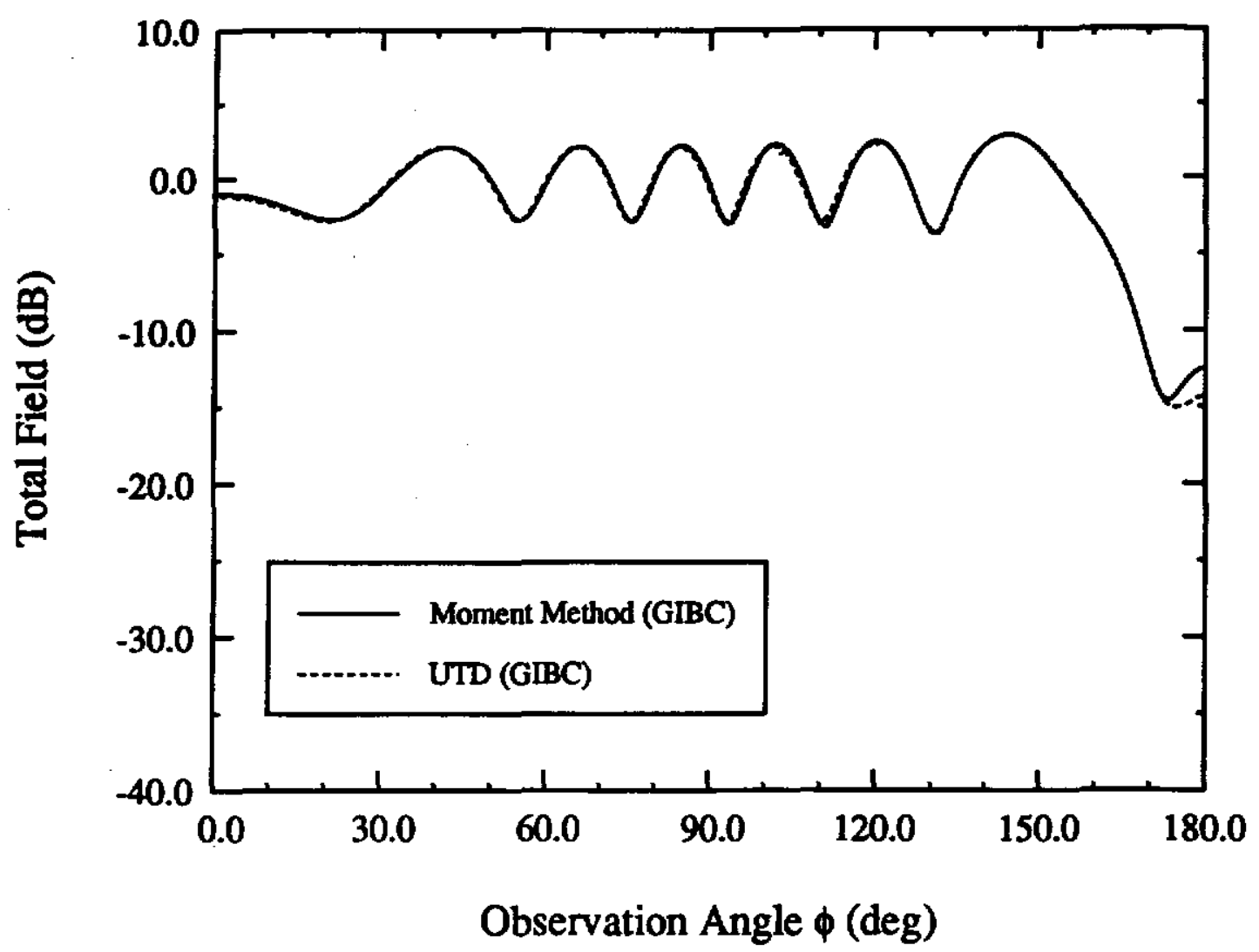

(a)

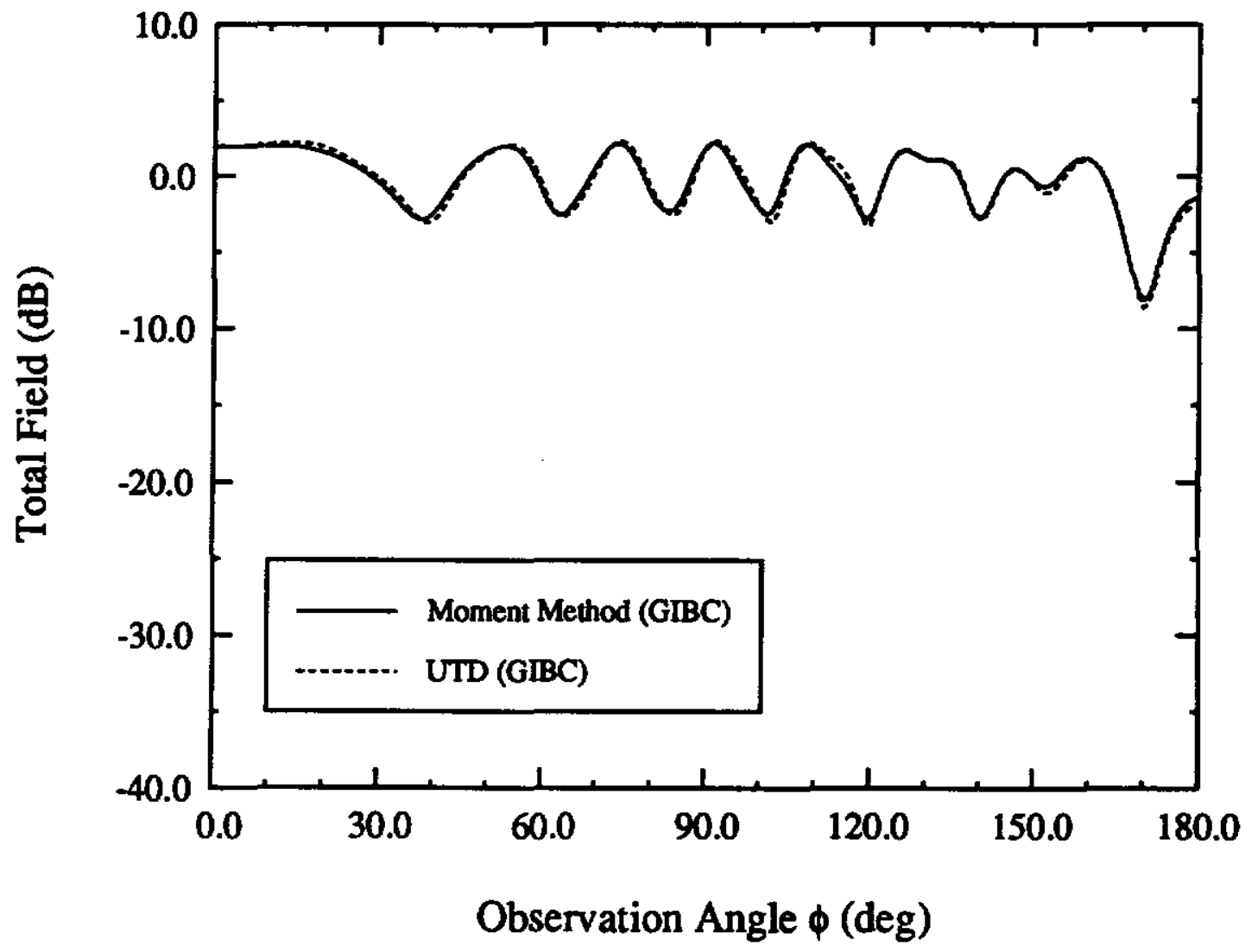

(b)

Fig. 9. Bistatic scattering pattern of an elliptical cylinder with $a=2 \lambda, b=1 \lambda, p=5 \lambda, \phi_{i}=0$ (a) E-polarization, $\varepsilon_{\mathrm{T}}=4, \mu_{\mathrm{T}}=1, \delta=0.07 \lambda$ (b) H-polarization, $\varepsilon_{\mathrm{T}}=8, \mu_{\mathrm{T}}=1, \delta=0.2 \lambda$. 


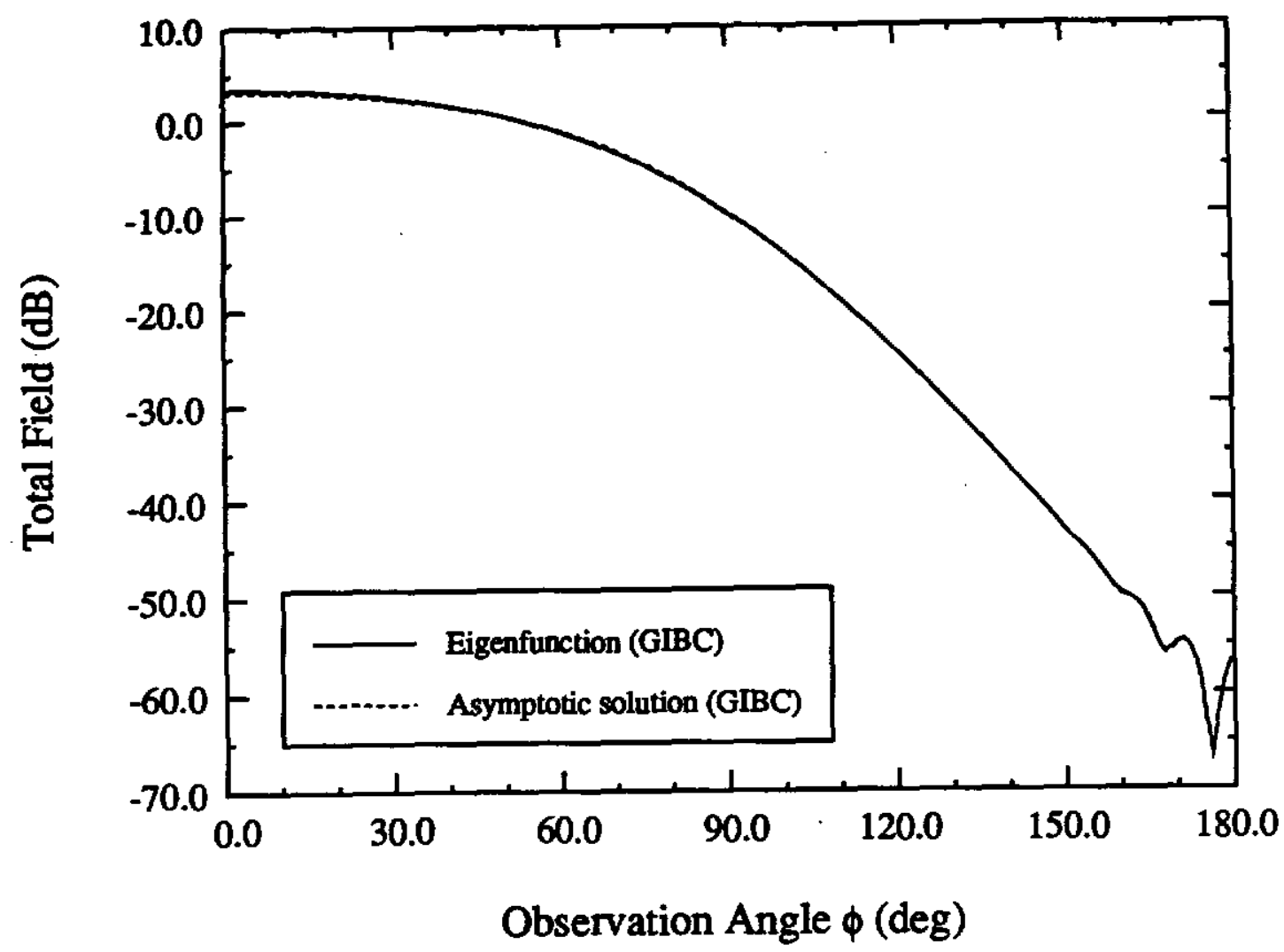

(a)

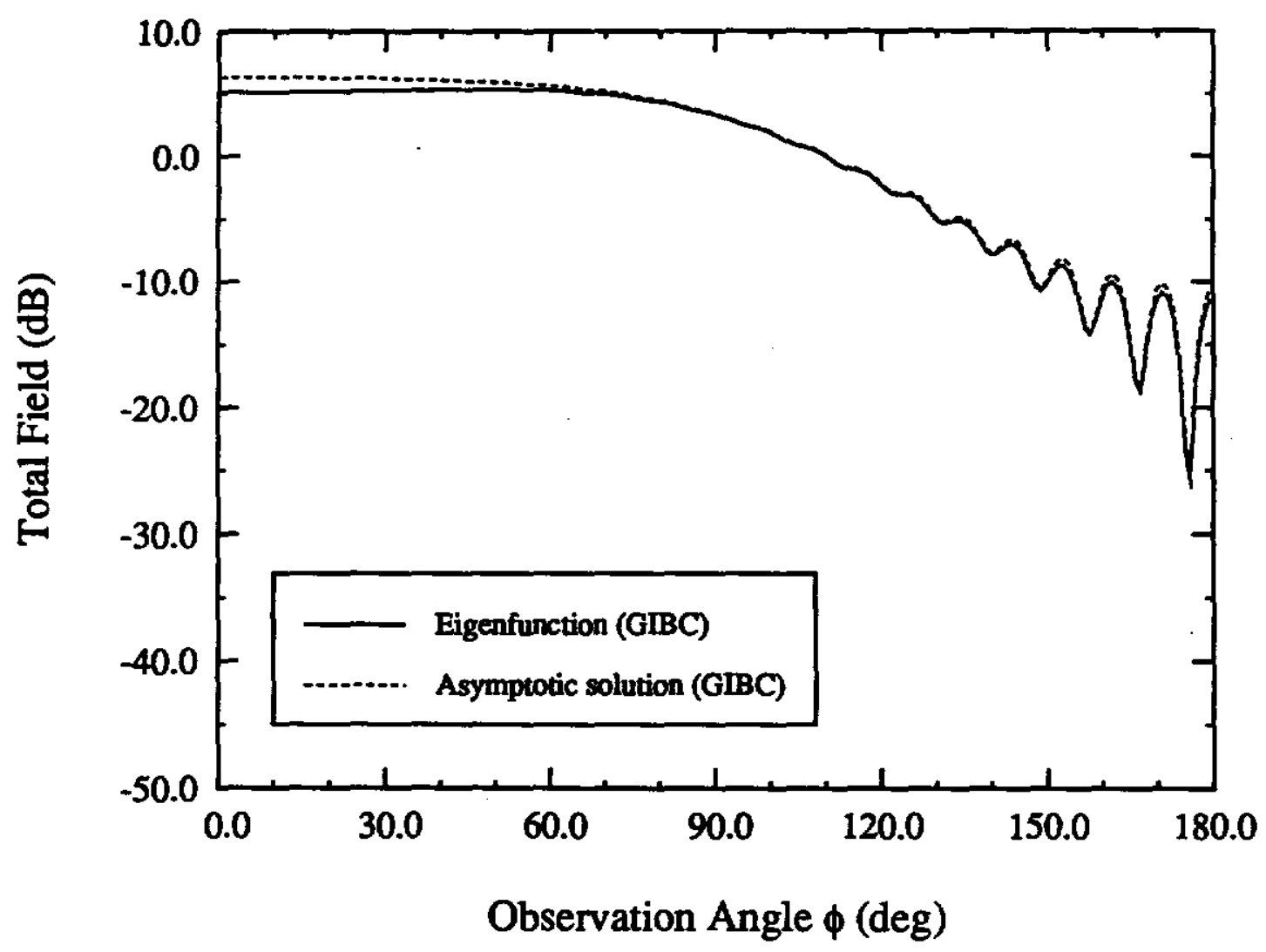

(b)

Fig. 10. Bistatic scattering pattern of a circular cylinder with $b=3 \lambda, \rho=3.05 \lambda, \phi_{i}=0$

(a) E-polarization, $\varepsilon_{\mathrm{T}}=4, \mu_{\mathrm{T}}=1, \delta=0.07 \lambda$ (b) H-polarization, $\varepsilon_{\mathrm{T}}=8, \mu_{\mathrm{T}}=1, \delta=0.2 \lambda$. 


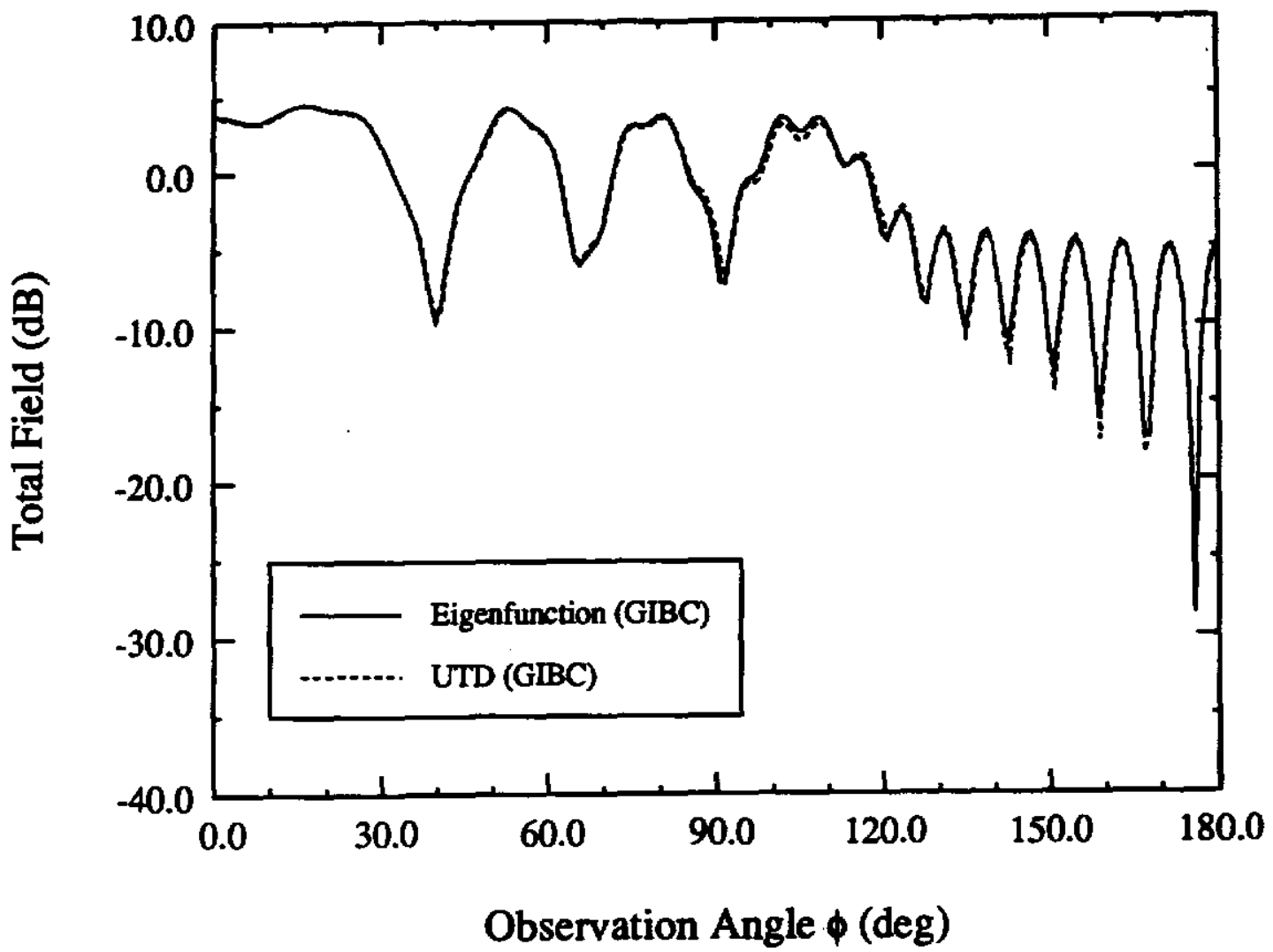

(a)

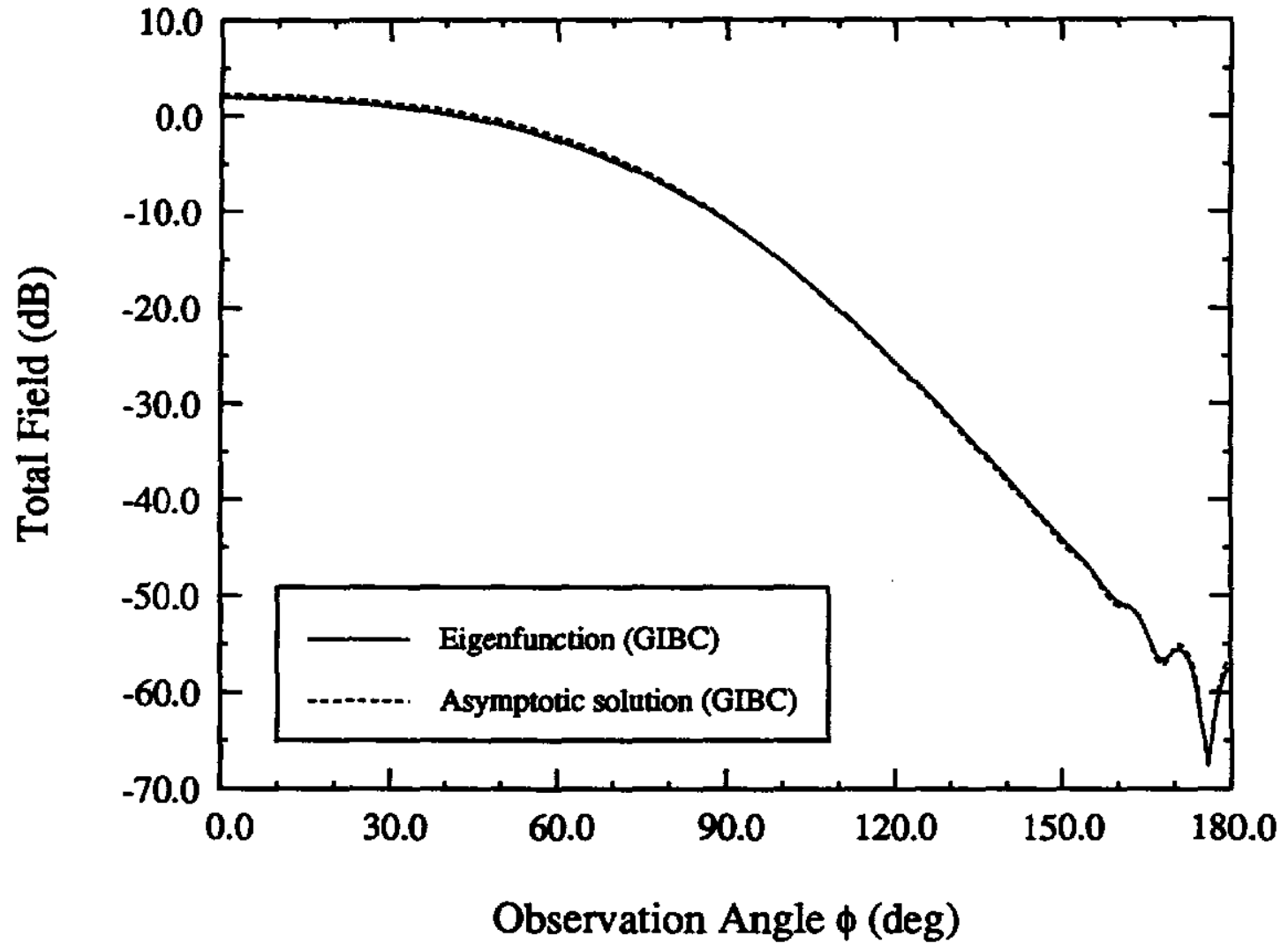

(b)

Fig. 11. Bistatic scattering pattern of a three-layer coated circular cylinder with $b=3 \lambda, \phi_{i}=0$, $\varepsilon_{\mathrm{T} 1}=3-\mathrm{j} 0.1, \varepsilon_{\mathrm{T} 2}=4-\mathrm{j} 0.3, \varepsilon_{\mathrm{T} 3}=7-\mathrm{j} 1.5, \mu_{\mathrm{T} 1}=\mu_{\mathrm{T} 2}=\mu_{\mathrm{T} 3}=1, \delta_{1}=0.01 \lambda$, $\delta_{2}=0.02 \lambda, \delta_{3}=0.03 \lambda$ (a) H-polarization, $\rho=5 \lambda$ (b) E-polarization, $\rho=3.05 \lambda$. 